Pacific Journal of Mathematics

ALGEBRAIC EQUIVALENCE OF LOCALLY NORMAL 


\title{
ALGEBRAIC EQUIVALENCE OF LOCALLY NORMAL REPRESENTATIONS
}

\author{
MASAMICHI TAKESAKI
}

\begin{abstract}
It will be shown that (i) the absolute value of every locally normal linear functional is again locally normal; (ii) two locally normal representations $\pi_{1}$ and $\pi_{2}$ of $\mathscr{A}$ generate isomorphic von Neumann algebras $\mathscr{M}\left(\pi_{1}\right)$ and $\mathscr{M}\left(\pi_{2}\right)$ if and only if there exists an automorphism $\sigma$ of $\mathscr{A}$ such that $\pi_{1} \circ \sigma$ and $\pi_{2}$ are quasi-equivalent, provided that either $\mathscr{M}\left(\pi_{1}\right)$ or $\mathscr{M}\left(\pi_{2}\right)$ is $\sigma$ finite.
\end{abstract}

This paper is motivated by a recent work [6] of R. Haag. R. V. Kadison and D. Kastler. As they mentioned, the recent progress in mathematical physics has made a precise analysis of representations of a $C^{*}$-algebra furnished with a net of von Neumann algebras a growing necessity.

In the first half of this paper, we shall show that the space of all locally normal linear functionals of a $C^{*}$-algebra with a net of von Neumann algebras is a closed invariant subspace of the conjugate space in the sense of [14], which will imply that the absolute value of a locally normal linear functional is locally normal too.

The last half of this paper will be devoted to extending a result of Powers [11] for UHF algebra to a $C^{*}$-algebra $\mathscr{A}$ with a proper sequential type $I_{\infty}$ funnel. Namely it will be shown that two locally normal representations $\pi_{1}$ and $\pi_{2}$ of the $C^{*}$-algebra $\mathscr{A}$ generate isomorphic von Neumann algebras if and only if they are connected by an automorphism of $\mathscr{A}$. This is proven under the assumption that one of the generated von Neumann algebras is $\sigma$-finite.

2. The locally normal conjugate space of a $C^{*}$-algebra with a net of von Neumann algebras. Let $\mathscr{A}$ be a $C^{*}$-algebra. Suppose a system $\mathfrak{F}=\left(\mathscr{A}_{\alpha}\right\}$ of $C^{*}$-subalgebras of $\mathscr{A}$ indexed by a directed set $\{\alpha\}$ is given such that:

(i) $\mathscr{A}_{\alpha}$ is a von Neumann subalgebra of $\mathscr{A}_{\beta}$ if $\alpha \leqq \beta$;

(ii) $\mathbf{U}_{\alpha} \mathscr{A}_{\alpha}$ is dense in $\mathscr{A}$ with respect to the norm topology. The system $\mathfrak{F}=\left\{\mathscr{A}_{\alpha}\right\}$ is called a net (in $\mathscr{A}$ ) of von Neumann algebras and each $\mathscr{A}_{\alpha}$ is called local subalgebra of $\mathscr{A}$

DEFINITION 1. A continuous linear functional $\varphi$ (resp. representation $\pi$ ) of $\mathscr{A}$ is said to be locally normal if $\varphi$ (resp. $\pi$ ) is $\sigma$-weakly continuous on each local subalgebra $\mathscr{A}_{\alpha}$. 
Proposition 2. Let $V$ be the set of all locally normal linear functionals on a $C^{*}$-algebra $\mathscr{A}$ with a net $\mathfrak{F}=\left\{\mathscr{A}_{\alpha}\right\}$ of von Neumann algebras. Then $V$ is a closed, invariant subspace of $\mathscr{A}^{*}$. Namely, if $\varphi \in \mathscr{A}^{*}$ is locally normal, then $a \varphi$ and $\varphi a, a \in \mathscr{A}$, are both locally normal, where a $\varphi$ and $\varphi$ a defined by $a \varphi(x)=\varphi(x a)$ and $\varphi a(x)=$ $\varphi(a x), x \in \mathscr{A}$.

Therefore, there exists a unique central projection $z_{0}$ of the universal enveloping von Neumann algebra $\widetilde{A}$ of $\mathscr{A}$, the second conjugate space of $\mathscr{A}$ as a Banach space, such that

$$
z_{0} \mathscr{A}^{*}=V \text {. }
$$

Proof. Let $\left\{\varphi_{n}\right\}$ be a sequence in $V$ converging to $\varphi \in \mathscr{A}^{*}$ with respect to the norm topology. For each $\alpha$, we have

$$
\left\|\left.\varphi\right|_{\mathscr{N}_{\alpha}}-\left.\varphi_{n}\right|_{\alpha_{\alpha}}\right\| \leqq\left\|\varphi-\varphi_{n}\right\| \rightarrow 0
$$

as $n \rightarrow \infty$; hence $\left\{\left.\varphi_{n}\right|_{\mathscr{N}_{\alpha}}\right\}$ converges to $\left.\varphi\right|_{\mathscr{N}_{\alpha}}$. Since the predual $\mathscr{A}_{\alpha^{*}}$ of each $\mathscr{A}_{\alpha}$ is complete, $\left.\varphi\right|_{\alpha_{\alpha}}$ belongs to $\mathscr{A}_{\alpha^{*}}$, so that $\phi$ is locally normal. Hence $V$ is closed.

Take an arbitrary element $\varphi \in V$. Let $a$ be an element of $\mathscr{A}_{\alpha}$. For each $\beta$, there exists an index $\gamma$ such that $\alpha \leqq \gamma, \beta \leqq \gamma$. Since $\left.\varphi\right|_{\mathscr{\sigma}_{\gamma}}$ is normal and $a$ is in $\mathscr{A}_{\gamma},\left.a \varphi\right|_{\mathscr{N}_{\gamma}}$ is normal, so that

$$
\left.a \varphi\right|_{\mathscr{A}_{\beta}}=\left.\left(\left.a \varphi\right|_{\mathscr{A}_{\gamma}}\right)\right|_{\varkappa_{\beta}}
$$

is normal. Hence $a \varphi$ belongs to $V$. Therefore, if $a$ belongs to $U \mathscr{A}_{\alpha}$, then $a \varphi$ is locally normal. If $a$ is an arbitrary element of $\mathscr{A}$, then there exists a sequence $\left\{a_{n}\right\}$ in $\mathbf{U} \mathscr{A}_{\alpha}$ such that

$$
\lim _{n \rightarrow \infty}\left\|a-a_{n}\right\|=0
$$

hence

$$
\lim _{n \rightarrow \infty}\left\|a \varphi-a_{n} \varphi\right\| \leqq \lim _{n \rightarrow \infty}\left\|a-a_{n}\right\|\|\varphi\|=0 .
$$

Therefore $a \varphi$ belongs to $V$ since $V$ is closed. By symmetry, $\varphi a$ is also in $V$. Hence $V$ is invariant.

The last half of our assertion follows from the fact that $V$ is invariant as a subspace of $\widetilde{\mathscr{A}}_{*}$ by [14]. This completes that proof.

As an immediate consequence of the above result, we get

CoRollary 3. In the same situation as Proposition 1, if $\varphi \in \mathscr{A}^{*}$ is locally normal, then the absolute value $|\varphi|$ of $\varphi$, in the sense of the polar decomposition, is locally normal too. In particular, if $\varphi \in \mathscr{A}^{*}$ is locally normal and self-adjoint, then the positive part $\varphi^{+}$ 
and the negative part $\varphi^{-}$of $\varphi$ are both locally normal.

This generalizes a result [6; Proposition 6] of Haag, Kadison and Kastler.

Proposition 4. In the same situation as before, $V$ is weak* sequencially complete. That is, if $\phi$ is a weak* limit of a sequence $\left\{\varphi_{n}\right\}$ of locally normal linear functionals, then $\varphi$ is locally normal too.

This follows directly from the weak sequential completeness of the predual $\mathscr{A}_{\alpha *}$ of each $\mathscr{A}_{\alpha}$, see for example [12].

3. Algebraic equivalence of locally normal representations. First of all, we recall the definition of algebraic equivalence of two representations given by Powers [11]:

Definition 5. Let $\left(\pi_{1}, \mathscr{H}_{1}\right)$ and $\left(\pi_{2}, \mathscr{H}_{2}\right)$ be two representations of a $C^{*}$-algebra $\mathscr{A}$. If the von Neumann algebras $\mathscr{C}\left(\pi_{1}\right)$ and $\mathscr{C}\left(\pi_{2}\right)$ generated by $\pi_{1}(\mathscr{A})$ and $\pi_{2}(\mathscr{A})$ respectively are isomorphic, then $\pi_{1}$ and $\pi_{2}$ are said to be algebraically equivalent.

The following is a slight modification of a definition given by Haag, Kadison and Kastler [6].

Definition 6. A sequential type $I_{\infty}$ funnel $\left\{\mathscr{A}_{n}\right\}$ of a $C^{*}$-algebra $\mathscr{A}$ is said to be proper if each relative commutant $\mathscr{A}_{n}^{\prime} \cap \mathscr{A}_{n+1}$ of $\mathscr{A}_{n}$ in $\mathscr{A}_{n+1}$ is of type $I_{\infty}$.

The following lemma is a modification of Glimm and Kadison's result [3].

Lemma 7. Let $\mathscr{C l}$ be a von Neumann algebra generated by an increasing sequence $\left\{\mathscr{A}_{n}\right\}$ of $C^{*}$-algebras, each of which contains the identity 1 of $\mathscr{C}$. Let $\mathscr{\mathscr { C }}\left(\mathscr{A}_{n}\right)$ denote the group of all unitary operators of $\mathscr{A}_{n}$. Then the union $\bigcup_{n=1}^{\infty} \mathscr{U}\left(\mathscr{A}_{n}\right)$ is strongly dense in the group $\mathscr{C}(\mathscr{L C})$ of unitary operators of $\mathscr{L}$.

Proof. Take an arbitrary unitary operator $u \in \mathscr{C}(M)$. There exists a self-adjoint operator $h \in \mathscr{C}$ such that $u=\exp (2 \pi i h)$ and $\|h\| \leqq 1$. Since $\bigcup_{n=1}^{\infty} \mathscr{A}_{n}$ is a strongly dense ${ }^{*}$-subalgebra of $\mathscr{A}$, there exists, by Kaplansky's density theorem [2: Th. 3, p. 43], a net $\left\{h_{j}\right\}_{j \in J}$ of self-adjoint elements in $\bigcup_{n=1}^{\infty} \mathscr{A}_{n}$ such that $\left\{h_{j}\right\}_{j \in J}$ converges strongly to $h$ and $\left\|h_{j}\right\| \leqq 1$. Put $u_{j}=\exp \left(2 \pi i h_{j}\right), j \in J$. Since each $h_{j}$ 
belongs to some $\mathscr{A}_{n}, u_{j}$ belongs to $\bigcup_{n=1}^{\infty} \mathscr{U}(\mathscr{A})$. By the strong continuity of the functional calculus on the bounded set of self-adjoint elements (see [10]), the net $\left\{u_{j}\right\}$ converges strongly to $u$. This completes the proof.

Lemma 8. Let $\mathscr{H}$ be a $\sigma$-finite (countably decomposable) von Neumann algebra. Suppose $\mathscr{A}$ and $\mathscr{B}$ are type $I_{\infty}$ subfactors of $\mathscr{M}$ with properly infinite relative commutants $\mathscr{A}^{\prime} \cap \mathscr{M}$ and $\mathscr{B}^{\prime} \cap \mathscr{M}$. Then there exists a unitary operator $u$ in $\mathscr{C}$ such that $u \mathscr{B} u^{-1}=\mathscr{A}$.

Proof. Let $\left\{u_{i, j}: i, j=1,2, \cdots\right\}$ and $\left\{v_{i, j}: i, j=1,2, \cdots\right\}$ be matrix units of $\mathscr{A}$ and $\mathscr{B}$ respectively. Put $e=u_{1,1}$ and $f=v_{1,1}$. Then $e$ and $f$ are minimal projections in $\mathscr{A}$ and $\mathscr{B}$ respectively. Since $\mathscr{A}^{\prime} \cap \mathscr{C}$ is properly infinite, $\mathscr{L}^{\prime} \cap \mathscr{C}$ contains an infinite sequence $\left\{p_{n}\right\}$ of equivalent orthogonal projections with $\sum p_{n}=1$. For each index $n$, let $u_{n}$ be a partial isometry in $\mathscr{A}^{\prime} \cap \mathscr{C}$ such that $u_{n}^{*} u_{n}=p_{1}$ and $u_{n} u_{n}^{*}=p_{n}$. Then we have

$$
\begin{aligned}
& \left(u_{n} e\right)^{*}\left(u_{n} e\right)=e u_{n}^{*} u_{n} e=e p_{1} ; \\
& \left(u_{n} e\right)\left(u_{n} e\right)^{*}=u_{n} e u_{n}^{*}=e p_{n} .
\end{aligned}
$$

Hence $\left\{e p_{n}\right\}$ is an infinite sequence of equivalent orthogonal projections with $\sum_{n=1}^{\infty} e p_{n}=e$, which means that $e$ is a properly infinite projection in $\mathscr{L}$. Similarly $f$ is a properly infinite projection in $\mathscr{M}$. Since $e$ and $f$ both have central support 1 , they are equivalent in $\mathscr{M}$, that is, there exists a partial isometry $w \in \mathscr{C}$ with $w^{*} w=e$ and $w w^{*}=f$ because of the $\sigma$-finiteness of $\mathscr{L}$. Put

$$
u=\sum_{i=1}^{\infty} v_{i, 1} w u_{1, i} \text {. }
$$

Then we have

$$
\begin{aligned}
u^{*} u & =\sum_{i, j=1}^{\infty} u_{i, 1} w^{*} v_{1, i} v_{j, 1} w u_{1, j} \\
& =\sum_{i=1}^{\infty} u_{i, 1} w^{*} w u_{1, i} \\
& =\sum_{i=1}^{\infty} u_{i, 1} u_{1,1} u_{1, i}=\sum_{i=1}^{\infty} u_{i, i} \\
& =1 ;
\end{aligned}
$$

similarly

$$
u u^{*}=1
$$

Hence $u$ is a unitary operator in $\mathscr{L}$. By a straightforward calculation, we have

$$
u u_{i, j} u^{*}=v_{i, j}, \quad i, j=1,2, \cdots
$$


hence we have

$$
u \mathscr{A} u^{*}=\mathscr{B}
$$

This completes the proof.

Lemma 9. Let $\mathscr{C l}$ be a $\sigma$-finite von Neumann algebra generated by a $C^{*}$-algebra $\mathscr{A}$ with a proper sequential type $I_{\infty}$ funnel $\left\{\mathscr{A}_{n}\right\}$, where we assume each $\mathscr{A}_{n}$ to be a von Neumann subalgebra of $\mathscr{A}$. Suppose $\mathscr{B}$ is a type $I_{\infty}$ factor contained in $\mathscr{A l}$ with properly infinite relative commutant $\mathscr{B}^{\prime} \cap \mathscr{M}$. For any $\sigma$-strong* neighborhood ${ }^{1)}$ $U$ of the identity 1 in $\mathscr{M}$, there exists $n$ and a unitary operator $u$ in $U$ such that

$$
u \mathscr{B} u^{-1} \subset \mathscr{A}_{n}
$$

Proof. By Lemma 8, there exists a unitary operator $v \in \mathscr{C}$ such that $v \mathscr{B} v^{-1}=\mathscr{A}_{1}$. Since $v^{-1}$ is in $\mathscr{A}$, it follows from Lemma 7 that there exists a unitary operator $w \in \mathscr{A}_{n}$ such that $w \in U v^{-1}$. Put $u=$ $w v$. Then $u$ belongs to $U$ and

$$
u \mathscr{B} u^{-1}=w v \mathscr{B} v^{-1} w^{-1}=w \mathscr{A}_{1} w^{-1} \subset \mathscr{A}_{n} .
$$

This completes the proof.

Lemma 10. Suppose $\mathscr{L}, \mathscr{A}$ and $\left\{\mathscr{A}_{n}\right\}$ are as in Lemma 9. Suppose $\mathscr{B}$ and $\mathscr{B}_{1}$ are both type $I_{\infty}$ subfactors of $M$ such that $\mathscr{B} \subset \mathscr{P}_{1}$ and the relative commutant $\mathscr{B}_{1}^{\prime} \cap \mathscr{L l}$ is properly infinite. Suppose $u$ is a unitary operator in $\mathscr{C}$ such that

$$
u \mathscr{B} u^{-1} \subset \mathscr{A}_{n_{0}} \cdot
$$

For any $\sigma$-strong* neighborhood $U$ of 1 in $\mathscr{A}$, there exist a unitary operator $u_{1}$ and index $n_{1}>n_{0}$ such that

(i ) $u_{1} \mathscr{B} u_{1}^{-1} \subset \mathscr{A}_{n_{1}}$;

(ii) $u_{1} x u_{1}^{-1}=u x u^{-1}$ for every $x \in \mathscr{B}$;

(iii) $u_{1} u^{-1} \in U$.

Proof. Put $\mathscr{C}=u \mathscr{B} u^{-1}$ and $\mathscr{C}_{1}=u \cdot \mathscr{B}_{1} u^{-1}$. Then $\mathscr{C}$ and $\mathscr{C}_{1}$ are both type $I_{\infty}$ subfactors of $\mathscr{C}$ with properly infinite relative commutant in $\mathscr{C}$. Put $\mathscr{N}=\mathscr{C}^{\prime} \cap \mathscr{M}$. Since $\mathscr{C}$ is a type $I$ subfactor of $\mathscr{K}, \mathscr{C}$ is decomposed into the tensor product:

1) The $\sigma$-strong* topology in a von Neumann algebra $\mathscr{M}$ is defined as the locally convex topology induced by the family of seminorms: $x \in \mathscr{K} \rightarrow p_{\omega}(x)=\omega\left(x^{*} x+x x^{*}\right)^{1 / 2}$, where $\omega$ runs over all normal states of $\mathscr{M}$. The $\sigma$-strong* topology agree with the strong operator topology on the unitary group of $\mathscr{M}$, but their uniform structures are different. 


$$
\mathscr{M} \cong \mathscr{C} \otimes\left(\mathscr{C}^{\prime} \cap \mathscr{M}\right)
$$

If $\mathscr{A}_{n} \supset \mathscr{C}$, then $\mathscr{A}_{n}$ is also decomposed with respect to this tensor product:

$$
\mathscr{A}_{n} \cong \mathscr{C} \otimes\left(\mathscr{C}^{\prime} \cap \mathscr{A}_{n}\right)
$$

Since $\mathrm{U} \mathscr{A}_{n}$ generates $\mathscr{C}, \mathrm{U}_{n=1}^{\infty}\left(\mathscr{C}^{\prime} \cap \mathscr{A}_{n}\right)$ generates $\mathscr{C}^{\prime} \cap \mathscr{M}=\mathscr{N}$. Let $\mathscr{D}$ be the uniform closure of $\bigcup_{n=1}^{\infty}\left(\mathscr{C}^{\prime} \cap \mathscr{A}_{n}\right)$. Then $\mathscr{D}$ has a sequential type $I$ funnel $\left\{\mathscr{C}^{\prime} \cap \mathscr{A}_{n}\right\}$. Since

$$
\mathscr{C}=u \mathscr{B} u^{-1} \subset \mathscr{A}_{n_{0}}
$$

by assumption, $\mathscr{C}^{\prime} \cap \mathscr{A}_{n}$ is properly infinite for $n>n_{0}$ because

$$
\mathscr{C}^{\prime} \cap \mathscr{A}_{n} \supset \mathscr{A}_{n} \cap \mathscr{A}_{n_{0}^{\prime}}^{\prime} \text {. }
$$

Moreover, we have, for $n>n_{0}$,

$$
\begin{aligned}
\left(\mathscr{C}^{\prime} \cap \mathscr{X}_{n}\right)^{\prime} \cap\left(\mathscr{C}^{\prime} \cap \mathscr{A}_{n+1}\right) \supset \mathscr{C}^{\prime} \cap \mathscr{A}_{n}^{\prime} \cap \mathscr{A}_{n+1} \\
\\
\supset \mathscr{A}_{n_{0}}^{\prime} \cap \mathscr{A}_{n}^{\prime} \cap \mathscr{A}_{n+1}=\mathscr{A}_{n}^{\prime} \cap \mathscr{X}_{n+1},
\end{aligned}
$$

hence $\left(\mathscr{C}^{\prime} \cap \mathscr{A}_{n}\right)^{\prime} \cap\left(\mathscr{C}^{\prime} \cap \mathscr{A}_{n+1}\right)$ is properly infinite. Hence the type $I_{\infty}$ funnel $\left\{\mathscr{C}^{\prime} \cap \mathscr{A}_{n}\right\}_{n>n_{0}}$ of $\mathscr{D}$ is proper. Put $\mathscr{D}_{1}=\mathscr{C}^{\prime} \cap \mathscr{C}_{1}$. Then $\mathscr{D}_{1}$ is a type $I_{\infty}$ subfactor of $\mathscr{N}$. Since

$$
\begin{aligned}
\mathscr{D}_{1}^{\prime} \cap \mathscr{N} & =\left(\mathscr{C}^{\prime} \cap \mathscr{C}_{1}\right)^{\prime} \cap\left(\mathscr{C}^{\prime} \cap \mathscr{M}\right) \\
& \supseteqq \mathscr{C}_{1}^{\prime} \cap \mathscr{M}=u\left(\mathscr{B}_{1}^{\prime} \cap \mathscr{C}\right) u^{-1},
\end{aligned}
$$

$\mathscr{D}_{1}$ has a properly infinite relative commutant $\mathscr{D}_{1}^{\prime} \cap \mathscr{N}$. By Lemma 9, there exists a unitary operator $v \in U \cap \mathscr{N}$ and an index $n_{1}$ such that $v \mathscr{D}_{1} v^{-1} \subset\left(\mathscr{A}_{n_{1}} \cap \mathscr{C}^{\prime}\right) \subset \mathscr{A}_{n_{1}}$. Put $u_{1}=v u$. Then $u_{1}$ is in $\mathscr{C}$ and $u_{1} u^{-1}$ is in $U$. For each $x \in \mathscr{B}, u x u^{-1}$ is in $\mathscr{C}$; hence it commutes with $v$, so that

$$
u_{1} x u_{1}^{-1}=v\left(u x u^{-1}\right) v^{-1}=u x u^{-1}
$$

Since

$$
\begin{aligned}
u_{1}\left(\mathscr{B}^{\prime} \cap \mathscr{B}_{1}\right) u_{1}^{-1} & =v u\left(\mathscr{B}^{\prime} \cap \mathscr{B}_{1}\right) u^{-1} v \\
& =v\left(\mathscr{C}^{\prime} \cap \mathscr{C}_{1}\right) v^{-1} \\
& =v \mathscr{D} v^{-1} \subset \mathscr{A}_{n_{1}}
\end{aligned}
$$

we have

$$
\begin{aligned}
u_{1} \mathscr{B}_{1} u_{1}^{-1} & =u_{1}\left(\mathscr{B} \cup\left(\mathscr{B}^{\prime} \cap \mathscr{B}_{1}\right)\right)^{\prime \prime} u_{1}^{-1} \\
& =\left(u_{1} \mathscr{B} u_{1}^{-1} \cup u_{1}\left(\mathscr{B}^{\prime} \cap \mathscr{B}_{1}\right) u_{1}^{-1}\right)^{\prime \prime} \\
& =\left(\mathscr{C} \cup v \mathscr{D} v^{-1}\right)^{\prime \prime} \subset\left(\mathscr{A}_{n_{0}} \cup \mathscr{A}_{n_{1}}\right)^{\prime \prime}=\mathscr{A}_{n_{1}} .
\end{aligned}
$$

This completes the proof. 
Lemma 11. Suppose $\mathscr{M}, \mathscr{A}$ and $\left\{\mathscr{A}_{n}\right\}$ are as in Lemma 9. Suppose $\mathscr{B}$ is another $C^{*}$-subalgebra of $\mathscr{C}$ with a proper sequential type $I_{\infty}$ funnel $\left\{\mathscr{B}_{n}\right\}$, which is $\sigma$-weakly dense in $\mathscr{L}$. Then there exists a unitary operator $u \in \mathscr{M}$ such that

$$
u\left(\bigcup_{n=1}^{\infty} \mathscr{A}_{n}\right) u^{-1}=\bigcup_{n=1}^{\infty} \mathscr{B}_{n}
$$

hence

$$
u \mathscr{A} u^{-1}=\mathscr{B}
$$

Proof. By the $\sigma$-finiteness of $\mathscr{C}$, there exists a faithful normal state $\varphi$ of $\mathscr{M}$. Define a distance function $d$ on $\mathscr{C}$ by:

$$
\left.d(x, y)=\left\{\varphi\left((x-y)^{*}(x-y)\right)+\varphi\left((x-y)(x-y)^{*}\right)\right\}\right\}^{1 / 2}, \quad x, y \in \mathscr{C l} .
$$

Then the topology induced by the metric $d$ coincides with the $\sigma$-strong* topology on the bounded part of $\mathscr{C l}$. Furthermore, the group $\mathscr{C}$ of all unitary operators of $\mathscr{C}$ is complete with respect to this metric $d$.

By induction, we construct increasing sequences $\left\{\mathscr{C}_{i}\right\},\left\{\mathscr{D}_{i}\right\}$ of type $I_{\infty}$ subfactors of $\mathscr{H}$, a sequence $\left\{u_{i}: i=1,2, \cdots\right\}$ of unitary operators in $\mathscr{C l}$ and increasing sequences $\left\{n_{i}\right\}$ and $\left\{m_{i}\right\}$ of integers with the properties:

$$
\begin{aligned}
& \mathscr{A}_{n_{i}+1} \subset \mathscr{C}_{i+1} \subset \mathscr{A}_{n_{i+1}} ; \\
& \mathscr{B}_{m_{i}+1} \subset \mathscr{D}_{i} \subset \mathscr{B}_{m_{i+1}}
\end{aligned}
$$

for $i=1,2, \cdots, k$;

$$
\begin{aligned}
& u_{2 i-1} \cdot \mathscr{B}_{m_{i}+1} u_{2 i-1}^{-1}=\mathscr{C}_{i}, \\
& u u_{2 i}^{-1} \mathscr{A}_{n_{i}+1} u_{2 i}=\mathscr{D}_{i}
\end{aligned}
$$

for $i=1,2, \cdots, k$;

$$
\begin{aligned}
& u_{2 i}^{-1} x u_{2 i}=u_{2 i-1}^{-1} x u_{2 i-1} \text { for } x \in \mathscr{C}_{i}, \\
& u_{2 i+1} x u_{2 i+1}^{-1}=u_{2 i} x u_{2 i}^{-1} \text { for } x \in \mathscr{D}_{i}
\end{aligned}
$$

for $i=1,2, \cdots, k$;

$$
d\left(u_{i}, u_{i+1}\right)<1 / 2^{i}
$$

for $i=1,2, \cdots, 2 k$.

For $k=1$, we choose $m_{1}=0, n_{1}=1$ and $\mathscr{C}_{1}=\mathscr{A}_{1}$. Then by Lemma 8 , there exists a unitary operator $u_{1}$ such that

$$
u_{1} \cdot \mathscr{B}_{1} u_{1}^{-1}=u_{1} \cdot \mathscr{B}_{m_{1}+1} u_{1}^{-1}=\mathscr{C}_{1}=\mathscr{A}_{1} \subseteq \mathscr{A}_{n_{1}} .
$$

Consider the triplet $\left(\mathscr{C}_{1}, \mathscr{A}_{2}, u_{1}^{-1}\right)$ as $\left(\mathscr{B}, \mathscr{B}_{1}, u\right)$ in Lemma 10 . Then 
we can find a unitary operator $v \in \mathscr{C}$ and index $m_{2}$ such that

$$
\begin{gathered}
v \mathscr{A}_{n_{1}+1} v^{-1}=v \mathscr{A}_{2} v^{-1} \subset \mathscr{B}_{m_{2}} ; \\
v x v^{-1}=u_{1}^{-1} x u_{1} \text { for every } x \in \mathscr{C}_{1} ; \\
d\left(u_{1}^{-1}, v\right)<\frac{1}{2} .
\end{gathered}
$$

Put $u_{2}=v$ and $\mathscr{D}_{1}=v \mathscr{A}_{2} v^{-1}$.

Suppose $\left\{n_{1}, \cdots, n_{k}\right\},\left\{m_{1}, \cdots, m_{k}\right\},\left\{\mathscr{C}_{1}, \cdots, \mathscr{C}_{k}\right\},\left\{\mathscr{D}_{1}, \cdots, \mathscr{D}_{k}\right\}$ and $\left\{u_{1}, \cdots, u_{2 k}\right\}$ have been chosen so that condition (i), (ii), (iii) and (iv) are satisfied. Applying Lemma 10 to $\left\{\mathscr{D}_{k}, \mathscr{B}_{m_{k}+1}, u_{2 k}\right\}$, we can find an index $n_{k+1}$ and a unitary operator $u_{2 k+1}$ such that

$$
\begin{gathered}
u_{2 k+1} \mathscr{B}_{m_{k}+1} u_{2 k+1}^{-1} \subset \mathscr{A}_{n_{k+1}} ; \\
u_{2 k+1} x u_{2 k+1}^{-1}=u_{2 k} x u_{2 k}^{-1} \text { for } x \in \mathscr{D}_{k} ; \\
d\left(u_{2 k}, u_{2 k+1}\right)<1 / 2^{2 k+1} .
\end{gathered}
$$

Put $\mathscr{C}_{k+1}=u_{2 k+1} \cdot \mathscr{B}_{m_{k}+1} u_{2 k+1}^{-1}$. Since

$$
u_{2 k} \mathscr{D}_{k} u_{2 k}^{-1}=\mathscr{A}_{n_{k}+1}
$$

$\mathscr{C}_{k+1}$ contains $\mathscr{A}_{n_{k}+1}$. Now again applying Lemma 10 to the triplet $\left\{\mathscr{C}_{k+1}, \mathscr{A}_{n_{k+1}+1}, u_{2 k+1}^{-1}\right\}$, we can choose an index $m_{k+1}$ and a unitary operator $u_{2(k+1)}$ in $\mathscr{C}$ such that

$$
\begin{gathered}
u_{2(k+1)}^{-1} \mathscr{A}_{n_{k+1}+1} u_{2(k+1)} \subset \mathscr{B}_{m_{k+1}} ; \\
u_{2(k+1)}^{-1} x u_{2(k+1)}=u_{2 k+1}^{-1} x u_{2 k+1} \text { for } x \in \mathscr{C}_{k+1} ; \\
d\left(u_{2 k+1}, u_{2(k+1)}\right)<1 / 2^{2(k+1)} .
\end{gathered}
$$

Put $\mathscr{D}_{k+1}=u_{2(k+1)}^{-1} \mathscr{C}_{n_{k+1}+1} u_{2(k+1)}$.

Hence the existence of sequences $\left\{m_{i}\right\},\left\{n_{i}\right\},\left\{\mathscr{C}_{i}\right\},\left\{\mathscr{D}_{i}\right\}$ and $\left\{u_{i}\right\}$ has been established. From condition (i) it follows that

$$
\bigcup_{i=1}^{\infty} \mathscr{A}_{i}=\bigcup_{i=1}^{\infty} \mathscr{C}_{i} \text { and } \bigcup_{i=1}^{\infty} \mathscr{B}_{i}=\bigcup_{i=1}^{\infty} \mathscr{D}_{i} \text {. }
$$

From condition (iv), $\left\{u_{k}\right\}$ is a Cauchy sequence of unitary operators with respect to the metric $d$. Hence $u_{k}$ converges $\sigma$-strongly* to a unitary operator $u$ of $\mathscr{l}$. By condition (iii), for every $x \in \mathscr{S}_{m_{i}+1}$, we have

$$
u_{k} v u_{k}^{-1}=u_{2 i-1} v u_{2 i-1}^{-1}
$$

for each $k \geqq 2 i$. Hence we have

$$
u \mathscr{B}_{m_{i}+1} u^{-1}=\mathscr{C}_{i} \text {. }
$$

Thus we have 


$$
\begin{aligned}
u\left(\bigcup_{k=1}^{\infty} \mathscr{B}_{k}\right) u^{-1} & =u\left(\bigcup_{j=1}^{\infty} \mathscr{B}_{m_{i}+1}\right) u^{-1} \\
& =\bigcup_{i=1}^{\infty} \mathscr{C}_{i}=\bigcup_{i=1}^{\infty} \mathscr{A}_{i} .
\end{aligned}
$$

This completes the proof.

As an immediate consequence of Lemma 11, we have the following extension of a corresponding result of Powers for UHF-algebras in [11].

TheOREM 12. Suppose $\mathscr{A}$ is a $C^{*}$-algebra with a proper sequencial type $I_{\infty}$ funnel $\left\{\mathscr{A}_{n}\right\}$. Suppose $\left\{\pi_{1}, \mathscr{H}_{1}\right\}$ and $\left\{\pi_{2}, \mathscr{H}_{2}\right\}$ are two locally normall representations of $\mathscr{A}$. Suppose either the von Neumann algebra $\mathscr{C}\left(\pi_{1}\right)$ generated by $\pi_{1}(\mathscr{A})$ or the one $\mathscr{C}\left(\pi_{2}\right)$ generated by $\pi_{2}(\mathscr{A})$ is $\sigma$-finite. Then the representations $\pi_{1}$ an $\pi_{2}$ are algebraically equivalent if and only if there exists an automorphism $\sigma$ of $\mathscr{A}$ such that the representations $\pi_{1}$ and $\pi_{2} \cdot \sigma$ are quasi-equivalent. If this is the case, then $\sigma$ may be chosen such that $\sigma\left(\bigcup_{n=1}^{\infty} \mathscr{A}_{n}\right)=\bigcup_{n=1}^{\infty} \mathscr{A}_{n}$.

Proof. If there exists an automorphism $\sigma$ of $\mathscr{A}$ such that $\pi_{1}$ and $\pi_{2} \circ \sigma$ are quasi-equivalent, then there exists an isomorphism $\rho$ of $\mathscr{C}\left(\pi_{1}\right)$ onto the von Neumann algebra $\mathscr{C}\left(\pi_{2} \circ \sigma\right)$ generated by $\pi_{2} \circ \sigma(\mathscr{A})$ such that $\rho \circ \pi_{1}=\pi_{2} \circ \sigma$. But it is clear that $\mathscr{C}\left(\pi_{2} \circ \sigma\right)=\mathscr{C}\left(\pi_{2}\right)$. Hence $\rho$ implements the algebraic equivalence of $\pi_{1}$ and $\pi_{2}$.

Suppose $\mathscr{C}\left(\pi_{1}\right)$ is $\sigma$-finite. Since $\mathscr{A}$ is simple (see [6: Proposition 10]), $\pi_{1}$ is an isomorphism of $\mathscr{A}$ into $\mathscr{C}\left(\pi_{1}\right)$. Hence we may identity $\mathscr{A}$ with the subalgebra $\pi_{1}(\mathscr{A})$ of $\mathscr{M}\left(\pi_{1}\right)$ which generates $\mathscr{L}\left(\pi_{1}\right)$. Suppose $\rho$ is an isomorphism of $\mathscr{C}\left(\pi_{2}\right)$ onto $\mathscr{L}\left(\pi_{1}\right)$. Put $\mathscr{B}=\rho \circ \pi_{2}(\mathscr{A})$ and $\mathscr{B}_{n}=\rho \circ \pi_{2}\left(\mathscr{A}_{n}\right)$. Then $\mathscr{C}\left(\pi_{1}\right), \mathscr{A},\left\{\mathscr{A}_{n}\right\}$ and $\mathscr{B}$, $\left\{\mathscr{B}_{n}\right\}$ satisfy all the assumptions of Lemma 11. Hence there exists a unitary operator $u$ in $\mathscr{C}\left(\pi_{1}\right)$ such that

$$
u \mathscr{A} u^{-1}=\mathscr{B}, u\left(\bigcup_{n=1}^{\infty} \mathscr{A}_{n}\right) u^{-1}=\bigcup_{n=1}^{\infty} \mathscr{B}_{n} .
$$

Define a map $\sigma$ of $\mathscr{A}$ into $\mathscr{A}$ by

$$
\sigma(x)=u^{-1} \rho \circ \pi_{2}(x) u,
$$

Then we have, for $x \in \mathscr{A}$,

$$
\pi_{1} \circ \sigma(x)=\sigma(x)=u\left(\rho \circ \pi_{2}(x)\right) u^{-1} ;
$$

hence $\pi_{1} \circ \sigma$ is unitary equivalent to $\rho \circ \pi_{2}$ and $\rho \circ \pi_{2}$ is quasi-equivalent to $\pi_{2}$ by definition, so that $\pi_{1} \circ \sigma$ and $\pi_{2}$ are quasi-equivalent. This completes the proof. 
CoRollary 13. If $\mathscr{A}$ is a $C^{*}$-algebra with a proper sequential type $I_{\infty}$ funnel, then the group of automorphisms of $\mathscr{A}$ acts transitively on the set of all locally normal pure states.

The author would like to express his thanks to Professor H. A. Dye for his kind hospitality at U. C. L. A. and he is indebted to Professor Herman who called his attention to the work [6] Haag, Kadison and Kastler.

\section{REFERENCES}

1. J. Dixmier, Les $C^{*}$-Algèbres et leurs représentations, Gauthier-Villars, Paris, 1964. 2. — Les algebres d'opérateurs dans l'espace Hilbertien, 2é edition, GauthierVillars, Paris, 1968.

3. J. Glimm and R. V. Kadison, Unitary operators in $C^{*}$-algebras, Pacific J. Math. 10 (1960), 547-556.

4. A. Guichardet, Algebres d'observables associes aux relations de commutation (to appear) 5. - Tensor products of $C^{*}$-algebras, Part II Infinite tensor product, Lecture notes, Aahus Univ., No. 12, 1969.

6. R. Haag, R. V. Kadison, and D. Kastler, Nets of $C^{*}$-algebras and classification of states (to appear)

7. R. Haag and D. Kastler, An algebraic approach to field theory, J. Math. Phys. 5 (1964), 848-861.

8. N. M. Hugenholtz, M. Takesaki, and M. Winnink, Local normality of the KMSstates in quantum statistical mechanics (in preparation)

9. N. M. Hugenholtz and J. D. Wieringa, On locally normal states in quantum statistical mechanics, Comm. Math. Phys. 11 (1969), 183-197.

10. I. Kaplansky, A theorem on rings of operators, Pacific J. Math. 1 (1951), 227-232.

11. R. T. Powers, Representations of uniformly hyper finite algebras and their associated von Neumann rings, Ann. of Math. 86 (1967), 138-171.

12. S. Sakai, The theory of $W^{*}$-algebras, mimeographed notes, Yale Univ., 1962.

13. Z. Takeda, Inductive limit and infinite direct product of operator algebras, Tôhoku Math. J. 7 (1955), 67-86.

14. M. Takesaki, On the conjugate space of operator algebra, Tôhoku Math. J. 10 (1958), 194-203.

Received January 22, 1970.

University of California, Los ANGeles, and

TôHoku University, SendaI 


\section{PACIFIC JOURNAL OF MATHEMATICS}

\section{EDITORS}

H. SAMELSON

Stanford University

Stanford, California 94305

\section{Richard Pierce}

University of Washington

Seattle, Washington 98105
J. DugundJI

Department of Mathematics

University of Southern California

Los Angeles, California 90007

RICHARD ARENS

University of California

Los Angeles, California 90024

\section{ASSOCIATE EDITORS}

\section{E. F. BeCKenBACH}

B. H. NeUmanN
K. YosHida

\section{SUPPORTING INSTITUTIONS}

\author{
UNIVERSITY OF BRITISH COLUMBIA \\ CALIFORNIA INSTITUTE OF TECHNOLOGY \\ UNIVERSITY OF CALIFORNIA \\ MONTANA STATE UNIVERSITY \\ UNIVERSITY OF NEVADA \\ NEW MEXICO STATE UNIVERSITY \\ OREGON STATE UNIVERSITY \\ UNIVERSITY OF OREGON \\ OSAKA UNIVERSITY \\ UNIVERSITY OF SOUTHERN CALIFORNIA
}

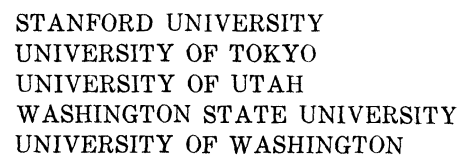

STANFORD UNIVERSITY UNIVERSITY OF TOKYO

UNIVERSITY OF UTAH

WASHINGTON STATE UNIVERSITY

UNIVERSITY OF WASHINGTON

The Supporting Institutions listed above contribute to the cost of publication of this Journal, but they are not owners or publishers and have no responsibility for its content or policies.

Mathematical papers intended for publication in the Pacific Journal of Mathematics should be in typed form or offset-reproduced, (not dittoed), double spaced with large margins. Underline Greek letters in red, German in green, and script in blue. The first paragraph or two must be capable of being used separately as a synopsis of the entire paper. The editorial "we" must not be used in the synopsis, and items of the bibliography should not be cited there unless absolutely necessary, in which case they must be identified by author and Journal, rather than by item number. Manuscripts, in duplicate if possible, may be sent to any one of the four editors. Please classify according to the scheme of Math. Rev. Index to Vol. 39. All other communications to the editors should be addressed to the managing editor, Richard Arens, University of California, Los Angeles, California, 90024.

50 reprints are provided free for each article; additional copies may be obtained at cost in multiples of 50 .

The Pacific Journal of Mathematics is published monthly. Effective with Volume 16 the price per volume (3 numbers) is $\$ 8.00$; single issues, $\$ 3.00$. Special price for current issues to individual faculty members of supporting institutions and to individual members of the American Mathematical Society: $\$ 4.00$ per volume; single issues $\$ 1.50$. Back numbers are available.

Subscriptions, orders for back numbers, and changes of address should be sent to Pacific Journal of Mathematics, 103 Highland Boulevard, Berkeley, California, 94708.

PUBLISHED BY PACIFIC JOURNAL OF MATHEMATICS, A NON-PROFIT CORPORATION

Printed at Kokusai Bunken Insatsusha (International Academic Printing Co., Ltd.), 7-17, Fujimi 2-chome, Chiyoda-ku, Tokyo, Japan. 


\section{Pacific Journal of Mathematics}

\section{Vol. 34, No. 3 \\ July, 1970}

Richard Hindman Bouldin, The peturbation of the singular spectrum

Hugh D. Brunk and Søren Glud Johansen, A generalized Radon-Nikodym derivative .

Henry Werner Davis, F. J. Murray and J. K. Weber, Families of $L_{p}$-spaces

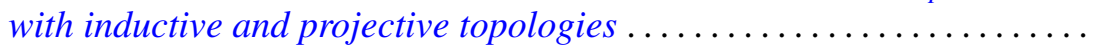

Esmond Ernest Devun, Special semigroups on the two-cell .

Murray Eisenberg and James Howard Hedlund, Expansive automorphisms

of Banach spaces ......................................

Frances F. Gulick, Actions of functions in Banach algebras.

Douglas Harris, Regular-closed spaces and proximities.

Norman Lloyd Johnson, Derivable semi-translation planes . .

Donald E. Knuth, Permutations, matrices, and generalized Young

tableaux..........................................

Herbert Frederick Kreimer, Jr., On the Galois theory of separable

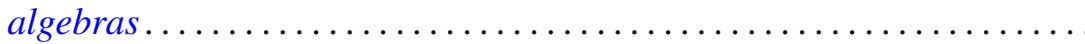

You-Feng Lin and David Alon Rose, Ascoli's theorem for spaces of

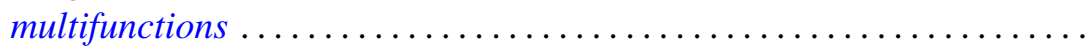

David London, Rearrangement inequalities involving convex functions . . . .

Louis Pigno, A multiplier theorem.

749

Helga Schirmer, Coincidences and fixed points of multifunctions into trees.

755

Richard A. Scoville, Some measure algebras on the integers .

Ralph Edwin Showalter, Local regularity of solutions of Sobolev-Galpern

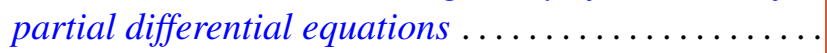

Allan John Sieradski, Twisted self-homotopy equivalences

John H. Smith, On S-units almost generated by S-units of subfields ...

803

Masamichi Takesaki, Algebraic equivalence of locally normal

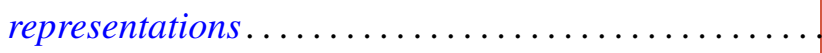

Joseph Earl Valentine, An analogue of Ptolemy's theorem and its converse in

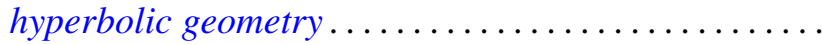

David Lawrence Winter, Solvability of certain p-solvable linear groups of finite order 